\title{
Papers
}

\section{Community pulmonary rehabilitation after hospitalisation for acute exacerbations of chronic obstructive pulmonary disease: randomised controlled study}

\author{
William D-C Man, Michael I Polkey, Nora Donaldson, Barry J Gray, John Moxham
}

\begin{abstract}
Objective To evaluate the effects of an early community based pulmonary rehabilitation programme after hospitalisation for acute exacerbations of chronic obstructive pulmonary disease (COPD).

Design A single centre, randomised controlled trial.

Setting An inner city, secondary and tertiary care hospital in London.

Participants 42 patients admitted with an acute exacerbation of COPD.

Intervention An eight week, pulmonary rehabilitation programme for outpatients, started within 10 days of hospital discharge, or usual care.

Main outcome measures Incremental shuttle walk distance, disease specific health status (St George's respiratory questionnaire, SGRQ; chronic respiratory questionnaire, CRQ) and generic health status (medical outcomes short form 36 questionnaire, SF-36) at three months after hospital discharge. Results Early pulmonary rehabilitation, compared with usual care, led to significant improvements in median incremental shuttle walk distance (60 metres, 95\% confidence interval 26.6 metres to 93.4 metres, $\mathrm{P}=0.0002)$, mean SGRQ total score $(-12.7,-5.0$ to $-20.3, \mathrm{P}=0.002)$, all four domains of the $\mathrm{CRQ}$ (dyspnoea 5.5, 2.0 to $9.0, \mathrm{P}=0.003$; fatigue $5.3,1.9$ to 8.8 , $\mathrm{P}=0.004$; emotion 8.7, 2.4 to $15.0, \mathrm{P}=0.008$; and mastery 7.5, 4.2 to $10.7, \mathrm{P}<0.001$ ) and the mental component score of the SF-36 (20.1, 3.3 to $36.8, \mathrm{P}=0.02)$. Improvements in the physical component score of the SF-36 did not reach significance (10.6, -0.3 to $21.6, \mathrm{P}=0.057$ ).

Conclusion Early pulmonary rehabilitation after admission to hospital for acute exacerbations of COPD is safe and leads to statistically and clinically significant improvements in exercise capacity and health status at three months.
\end{abstract}

\section{Introduction}

Admissions to hospital for acute exacerbations of chronic obstructive pulmonary disease (COPD) are a massive burden to the NHS. Over the past decade, such admissions have increased by $50 \%$, and annual hospital costs for COPD in the United Kingdom amount to more than $£ 587 \mathrm{~m}(\$ 1.05 \mathrm{bn} ; € 850 \mathrm{~m}) .{ }^{1}$ Exacerbations are also associated with impaired quality of life, reduced exercise capacity, and increased risk of readmission. ${ }^{2}$ Interventions designed to hasten recovery and improve symptoms after admission to hospital may lead not only to reduced use of health care in the future (and subsequent economic benefits to the
NHS) but also to real improvements in quality of life and functional ability in breathless and vulnerable patients with COPD.

Pulmonary rehabilitation is a multidisciplinary programme of care for patients with chronic respiratory impairment that is individually tailored and designed to optimise each patient's physical and social performance and autonomy. Pulmonary rehabilitation leads to statistically significant and clinically meaningful improvements in health related quality of life, functional exercise capacity, and maximum exercise capacity in patients with stable COPD. ${ }^{3}$ Consequently, the recent guidelines on the management of COPD published by the National Institute for Clinical Excellence (NICE) and the British Thoracic Society recommend that pulmonary rehabilitation should be made available to all appropriate patients. ${ }^{5}$ However, the effects of early pulmonary rehabilitation of outpatients in the acute recovery phase after hospital admission for acute exacerbations of COPD have not previously been studied. Patients are particularly vulnerable after a hospital admission, and we assumed that early pulmonary rehabilitation of outpatients would lead to notable improvements in exercise capacity and health status, as it does in stable patients with COPD.

We assessed the feasibility and safety of an early pulmonary rehabilitation programme for outpatients and determined the effects on exercise capacity and quality of life, compared with usual care, at three months after a hospital admission for acute exacerbation of COPD.

\section{Methods}

\section{Patients}

We recruited 42 patients admitted to King's College Hospital in London with a primary diagnosis of acute exacerbation of COPD. All were deemed sufficiently unwell by the duty medical registrar to warrant admission to hospital. Exclusion criteria included comorbidity that could limit exercise training. In addition, to allow for a suitable washout period, we also excluded patients who had attended a pulmonary rehabilitation programme in the preceding year. All patients gave written informed consent.

\section{Protocol}

All admitted patients received standard treatment, including nebulised bronchodilators, oxygen, oral or intravenous antibiot-

The CONSORT checklist of items to include when reporting a randomised controlled trial is on bmj.com 
Table 1 Patients' characteristics on day of hospital discharge. Values are means with standard deviations unless otherwise indicated

\begin{tabular}{lcc} 
& Usual care group $(\mathbf{n}=\mathbf{2 1})$ & Rehabilitation group $(\mathbf{n}=\mathbf{2 1})$ \\
\hline Age in years & $70.7(9.3)$ & $69.6(9.2)$ \\
\hline No of men (women) & $8(13)$ & $9(12)$ \\
\hline $\mathrm{FEV}_{1}(\%$ predicted) & $36.7(14.9)$ & $41.7(18.9)$ \\
\hline Length of stay in days & $8.8(4.3)$ & $8.0(3.9)$ \\
\hline $\begin{array}{l}\text { Incremental shuttle walk } \\
\text { distance in metres }\end{array}$ & $110(64)$ & $116(79)$ \\
\hline $\begin{array}{l}\text { Total score on St George's } \\
\text { respiratory questionnaire }\end{array}$ & $67.6(13.3)$ & $65.7(13.8)$ \\
\hline
\end{tabular}

Unpaired $t$ tests were used for comparisons between groups.

ics, non-invasive ventilation (if required), and a one to two week course of oral prednisolone (30-40 mg daily). On discharge from hospital, patients were allocated to either an early pulmonary rehabilitation programme (within 10 days of hospital discharge) or usual care. Both groups of patients were discharged having optimal medical treatment, with a summary of their admission, to the care of their general practitioner and received standard follow up outpatient appointments with a pulmonary specialist. All patients received home diaries, which included a disease specific information pack.

\section{Baseline assessment}

We made baseline assessments in the 24 hours before patients were discharged from hospital and assigned to the intervention. We measured exercise capacity by the incremental shuttle walk test $^{6}$ a standardised, externally paced, corridor walking test, which is reproducible after a single practice walk. Patients are instructed to walk along a 10 metre course in time with prerecorded signals; initial walking speed is set at 0.50 metres per second, and increased each minute by 0.17 metres/second. The distance walked correlates well with maximum consumption of oxygen. The incremental shuttle walk is commonly used in pulmonary rehabilitation programmes in the United Kingdom as a functional outcome measure of maximum exercise capacity. We used the St George's respiratory questionnaire (SGRQ) and the chronic respiratory disease questionnaire (CRQ), both well validated in patients with $\mathrm{COPD}^{78}$ and often used outcome measures in pulmonary rehabilitation studies, to measure disease specific health status. We measured generic health status with the short form, 36 item questionnaire for medical outcomes (SF-36). ${ }^{9}$

\section{Assignment}

A random number generator was our tool to assign an intervention to the first patient entering the study. We used the minimisation method to assign patients further to the intervention group, taking into account five factors: age ( $<70$ years or $\geq 70$ years), sex, length of hospital admission ( $<7$ days or $\geq 7$ days), incremental shuttle walk distance at discharge $(<100$ metres or $\geq 100$ metres), and predicted forced expiratory volume in one second $\left(\mathrm{FEV}_{1} ;<30 \%\right.$ or $\left.\geq 30 \%\right)$. Table 1 shows baseline characteristics.

\section{Pulmonary rehabilitation}

A multidisciplinary team ran the pulmonary rehabilitation programme, which consisted of two classes per week for eight weeks. Patients were given a choice of three locations around the London boroughs of Lambeth and Southwark, but the classes were run by the same team with the same equipment. Each class lasted two hours, consisting of one hour of exercise (aerobic walking and cycling, strength training for the upper and lower limb) and one hour of educational activities (with an emphasis on self management of the disease, nutrition, and lifestyle issues). Respiratory physiotherapists and nurses supervised the exercise component, as did health centre based fitness instructors. Physiotherapists, respiratory nurses, an occupational therapist, a dietician, a respiratory doctor, a smoking cessation adviser, a social worker, a pharmacist, and a lay member of a patients' group supervised education activities on a rolling rota. Patients also received individualised home exercise programmes, which encouraged at least 20 minutes of exercise per day.

\section{Assessment of outcome}

We reassessed primary outcome measures (exercise capacity and disease specific and generic health status) at three months after hospital discharge. In addition, we recorded secondary outcome measures of use of hospital resources, including number of readmissions, hospital days, and visits to the accident and emergency department not requiring admission. We gathered this information principally from hospital records, corroborated by the patients' home diaries.

\section{Blinding}

Owing to the nature of the intervention and financial and logistical considerations, it was not possible to blind the patients or the assessors. The assessors were either the investigator responsible for assignment or members of the pulmonary rehabilitation team who were directly or indirectly involved in the delivery of the intervention.

\section{Sample size calculation}

The primary outcome measure for the study was the incremental shuttle walk distance. On the basis of previous audit data, a sample size calculation estimated that at least 30 patients ( 15 in each group) would be required to show a 50 metre difference in shuttle walk distance (standardised difference 1.2) with $90 \%$ power at the $5 \%$ significance level.

\section{Data analysis and statistical methods}

We analysed data on an intention to treat basis. We made no attempt to impute "missing" data from those participants who were lost to follow up. We calculated means (standard deviations) or medians (ranges or interquartile ranges) as appropriate. We used unpaired $t$ tests to compare mean differences between groups for SGRQ, CRQ and SF-36 scores. Because of the highly skewed distribution we used Mann-Whitney and univariate median regressions to compare median shuttle walks between groups. We used the Mann-Whitney test to test differences between groups in hospital bed days. We used incident rate ratios and the Poisson regression for rates of hospital readmission and accident and emergency visits. We used SPSS, version 12, and Stata, version 8 , for the computations.

\section{Results}

\section{Flow and follow up of participants}

Figure 1 shows the progress of participants through the study. We initially assessed 69 patients for eligibility over a six month period. Seventeen patients were not enrolled for medical reasons or met exclusion criteria: coexisting unstable ischaemic heart disease $(n=7)$, pulmonary rehabilitation in preceding year $(\mathrm{n}=4)$, coexisting probable or definite diagnosis of lung cancer $(n=3)$, chronic alcohol abuse $(n=2)$, and wheelchair bound owing to rheumatoid arthritis $(\mathrm{n}=1)$. Ten patients refused consent: "felt too ill" $(\mathrm{n}=4)$, "clash with other social activities" $(\mathrm{n}=3)$, "no likely benefit to me" $(\mathrm{n}=3)$. Of the 42 patients recruited, three month exercise capacity and health status data were not available for eight patients because they died $(n=3)$, 


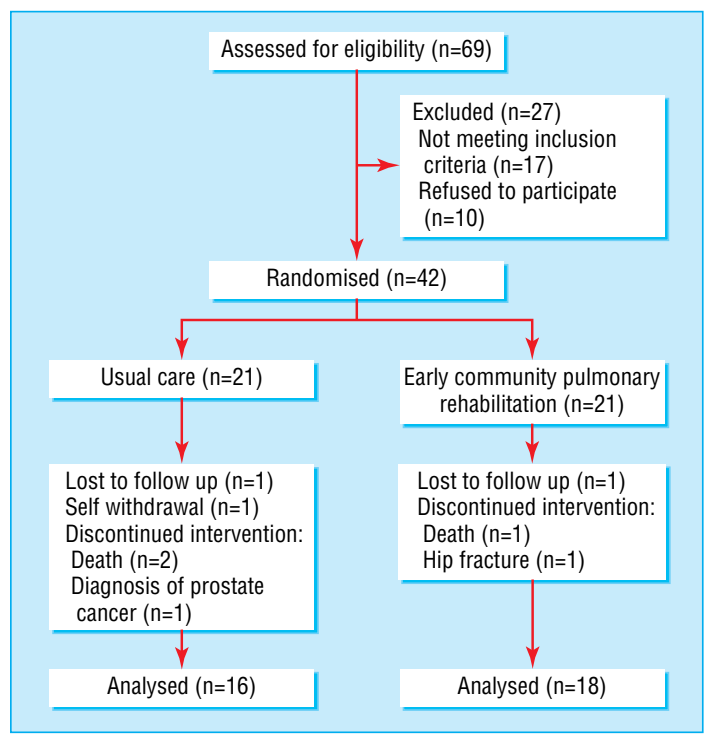

Flow of participants through the study

were lost to follow up because they had moved abroad or out of area $(n=2)$, developed serious comorbidity unrelated to COPD $(n=2)$, and withdrew because of allocation to usual care $(n=1)$. The mean attendance rate at the pulmonary rehabilitation class was $73 \%$, with 6 of the 18 patients attending less than $50 \%$ of the classes. No adverse events were reported in the early rehabilitation group. Table 2 shows baseline and three month data in the usual care and early pulmonary rehabilitation groups.

Exercise capacity, the SGRQ impacts and total score, all four domains of the CRQ, and the mental component score of the SF-36 in favour of early pulmonary rehabilitation improved significantly. The magnitude of these mean improvements greatly exceeded the recognised minimal clinically important differences for these measures. In addition, the rehabilitation group made far fewer visits to accident and emergency departments, and we saw a trend towards reduced hospital readmission rate and fewer hospital days.

\section{Discussion}

Attendance at a community based pulmonary rehabilitation programme shortly after a hospital admission for an acute exacerbation of COPD leads to clinically important improvements in exercise capacity and health status at three months. Furthermore, the number of attendances at the accident and emergency department was lower and hospital admission was required in fewer cases.

\section{Comparison with other studies}

Excellent evidence supports the benefits of pulmonary rehabilitation in stable patients with COPD. ${ }^{3} 4$ This study examines the effects of this outpatient intervention in patients during the early recovery period after a hospital admission for an acute exacerbation. Despite optimal medical treatment during hospital admission, patients at discharge take considerable time to recover to baseline levels of physical functioning and health status. Previous studies have shown that up to $25 \%$ of patients after an acute exacerbation do not fully recover to baseline peak flow at three months ${ }^{10}$ and that the recovery period in health status is long even in patients who do not have further exacerbations. ${ }^{11}$ Our data indicate that patients can safely participate in a community based pulmonary rehabilitation programme for outpatients shortly after an exacerbation and that such a programme speeds up recovery from the debilitating effects of a hospital admission. Furthermore, the magnitude of the effects of early pulmonary rehabilitation on exercise capacity and health

Table 2 Baseline and three month data. Data are mean scores (SD) and differences between groups were compared by using unpaired $t$ tests unless otherwise indicated

\begin{tabular}{|c|c|c|c|c|c|c|}
\hline \multirow[b]{2}{*}{ Outcome measure } & \multicolumn{2}{|c|}{ Usual care $(n=16)$} & \multicolumn{2}{|c|}{ Early rehabilitation $(n=18)$} & \multirow{2}{*}{$\begin{array}{l}\text { Mean difference between } \\
\text { groups }(95 \% \mathrm{Cl})\end{array}$} & \multirow[b]{2}{*}{$P$ value } \\
\hline & At discharge & At three months & At discharge & At three months & & \\
\hline \multicolumn{7}{|l|}{ Shuttle walk test in metres: } \\
\hline Median distance (range) & $115(20-190)$ & $90(10-250)$ & $120(10-330)$ & $210(40-400)$ & $60(26.6 \text { to } 93.4)^{*}$ & $0.0002 \dagger$ \\
\hline \multicolumn{7}{|c|}{ St George's respiratory questionnaire (range 100-0) $\ddagger:$} \\
\hline Symptoms & $77.7(14.6)$ & $76.8(15.4)$ & $73.1(18.2)$ & $69.1(16.7)$ & $-3.1(-12.1$ to 5.8$)$ & 0.48 \\
\hline Activities & $87.4(8.7)$ & $84.6(15.0)$ & $81.3(15.1)$ & $70.5(19.6)$ & $-8.1(-17.6$ to 1.5$)$ & 0.10 \\
\hline Impacts & $56.8(19.2)$ & $52.3(16.6)$ & $53.7(17.7)$ & $30.8(16.9)$ & $-18.4(-28.7$ to -8.1$)$ & 0.001 \\
\hline Total & $69.6(13.5)$ & $66.2(13.6)$ & $65.4(14.1)$ & $49.3(15.3)$ & $-12.7(-5.0$ to -20.4$)$ & 0.002 \\
\hline \multicolumn{7}{|c|}{ Chronic respiratory disease questionnaire§: } \\
\hline Dyspnoea (range 5-35) & $11.4(4.4)$ & $13.5(4.3)$ & $11.8(5.0)$ & $19.4(5.2)$ & 5.5 (2.0 to 9.0$)$ & 0.003 \\
\hline Fatigue (range 4-28) & $11.6(6.1)$ & $13.8(5.1)$ & $9.9(2.8)$ & $17.4(5.4)$ & 5.3 (1.9 to 8.8) & 0.004 \\
\hline Emotion (range 7-49) & $27.0(12.6)$ & $29.7(11.4)$ & $21.1(5.8)$ & $32.5(7.2)$ & 8.7 (2.4 to 15.0$)$ & 0.008 \\
\hline Mastery (range 4-28) & $13.6(7.4)$ & $16.1(7.0)$ & $10.8(4.8)$ & $20.7(5.0)$ & 7.5 (4.2 to 10.7) & $<0.001$ \\
\hline \multicolumn{7}{|l|}{ SF-36 (range 0-100)ף: } \\
\hline Physical component & $30.4(19.9)$ & $36.4(22.2)$ & $26.3(14.6)$ & $43.0(20.0)$ & $10.6(-0.3$ to 21.6$)$ & 0.057 \\
\hline Mental component & $41.6(21.9)$ & $47.2(24.2)$ & $30.6(14.2)$ & $56.3(24.0)$ & 20.1 (3.3 to 36.8) & 0.02 \\
\hline $\begin{array}{l}\text { Hospital readmissions rate } \\
\text { in \% }\end{array}$ & - & 57.1 & - & 35.0 & $0.66(0.3 \text { to } 1.5)^{* *}$ & $0.30+\dagger$ \\
\hline Total hospital days & - & 123 & - & 86 & - & $0.21 \dagger$ \\
\hline $\begin{array}{l}\text { Accident and emergency } \\
\text { visit rate in \% }\end{array}$ & - & 42.9 & - & 10.0 & $0.14(0.03 \text { to } 0.65)^{* *}$ & $0.01 \mathrm{t \dagger}$ \\
\hline
\end{tabular}

* Median difference.

†Mann-Whitney test.

$\ddagger$ Decreased score denotes improvement. Minimal clinically important difference is 4 points on the total score.

§Increased score denotes improvement. Minimal clinically important difference is 2.5 (dyspnoea domain), 2 (fatigue domain), 3.5 (emotion domain) and 2 (mastery domain).

Ilncreased score denotes improvement.

${ }^{* *}$ Incident rate ratio.

††Poisson regression. 
status are considerably greater than, and in addition to, the effects of bronchodilator or corticosteroid therapy. ${ }^{12}$

\section{Limitations of the study}

The study did not explore the mechanisms by which early pulmonary rehabilitation achieves its effects. Possible explanations include physiological improvements in skeletal muscle function, desensitisation to dyspnoea, and psychosocial lifestyle changes. Dysfunction of the skeletal muscles is well recognised in patients with $\mathrm{COPD}^{13}$ and is particularly marked during a hospital admission ${ }^{14}$ owing to a variety of factors, including atrophy resulting from disuse, systemic inflammatory mediators, and use of corticosteroids. A limitation of this study is that, for obvious reasons, it was not possible to blind patients to the intervention. Hence a placebo effect cannot be excluded as the principal mechanism for the improvements observed. However, it should be noted that early pulmonary rehabilitation led to considerable improvements in the distances covered in the incremental shuttle walk. The maximum distance walked during this test correlates well with peak oxygen consumption, ${ }^{6}$ hence it is likely that a true physiological training effect contributed to the improvements. The psychological effects, effects of self education about the disease, or placebo effects of pulmonary rehabilitation in an often socially isolated group of patients should not be minimised. A recent, multicentre, randomised controlled trial showed that an education programme for patients (consisting of weekly visits by a health professional for a two month period, with monthly telephone follow up) led to reduced use of healthcare resources in patients with severe COPD. ${ }^{15}$ Another limitation of the study is that the assessors were not fully blinded to treatment allocation as they may have been directly or indirectly involved in the delivery of the intervention. This introduces an element of bias to the results, but it is important to note that the SGRQ and the SF-36 questionnaires are completed by the patients themselves, without direct input from investigators, and the incremental shuttle walk and the CRQ are highly standardised outcome measures that do not place subjective requirements on the investigator.

\section{Viability of the programme}

Exercise training after an acute exacerbation of COPD is not a novel concept. Behnke et al looked at the effects of an initial, 10 day, inpatient training programme, followed by six months of supervised home training, compared with usual care, in patients admitted for an acute exacerbation of COPD. ${ }^{16}$ They showed improvements in six minute walking distance and sum scores on the questionnaire on chronic respiratory disease at three months and six months after training compared with control. However, such a programme would not be viable in terms of manpower or finance, given that inpatient stay contributes 54\% of all direct healthcare costs associated with COPD in the United Kingdom. ${ }^{17}$ In contrast, a community based pulmonary rehabilitation programme for outpatients is a more realistic option. Previous data support the cost effectiveness of pulmonary rehabilitation programmes on an outpatient basis and the likelihood of financial benefit to the health service. ${ }^{18}$

\section{Possible health economic impact and outlook}

The a priori primary outcome measures were exercise capacity, as measured by the incremental shuttle walk, and health status. However, secondary outcome measures included use of hospital resources, and fewer visits were made to the accident and emergency department in the group undergoing early pulmonary rehabilitation. Patients in the treated group were readmitted 30\% less often than patients in the control group, and there was a

\section{What is already known on this topic}

Hospital admission for acute exacerbations of chronic obstructive pulmonary disease (COPD) is an enormous financial burden to the NHS

Pulmonary rehabilitation is an effective, non-pharmacological intervention in the management of stable patients with COPD, and the recent guidelines from the National Institute for Clinical Excellence recommend that it should be made available to all patients who would benefit

\section{What this study adds}

Early pulmonary rehabilitation, in the recovery period after hospital discharge after an admission for an acute exacerbation of COPD, leads to significant improvements in functional capacity and quality of life at three months compared with usual care

trend towards fewer hospital inpatient days. The results therefore imply that early pulmonary rehabilitation may reduce usage of healthcare resources and bring improvements in exercise capacity and health status.

Larger randomised studies are required to determine whether the benefits of early pulmonary rehabilitation translate into improved health economics. Other unanswered questions include the long term effects of early pulmonary rehabilitation, and the optimal structure, location, and duration of pulmonary rehabilitation programmes.

Despite medical optimisation during hospital admission for acute exacerbations of COPD, early pulmonary rehabilitation after discharge from hospital leads to additional notable improvements in exercise capacity and health status at three months compared with usual care.

We thank the Southwark and Lambeth pulmonary rehabilitation team for running and supervising the rehabilitation programme described in this work.

Contributors: WD-CM conceived the idea of the study, obtained grant funding, designed the protocol, recruited subjects, collected and analysed data and wrote the first and final drafts of the manuscript. MIP helped design the protocol of the study, interpreted data, and helped write the first and final drafts of the manuscript. ND helped design the study, performed statistical analysis of the data and helped write the first and final drafts of the manuscript. BJG helped design the study, recruited patients, interpreted data and helped write the first and final drafts of the manuscript. JM was the senior investigator, and will act as guarantor. He helped conceive the idea and design of the study, obtained grant funding, interpreted data, and helped write the first and final drafts of the manuscript. Christine Jones and Caroline Mooney helped in the recruitment of subjects. Renata Morello, Sheena Radford, Julie Backley, Lynda Haggis, Claire Bradley, Lauren Moore, and Debbie James were involved in data collection and were members of the multidisciplinary team that provided the active intervention.

Funding: This study was supported by a British Lung Foundation Trevor Clay Memorial Grant. WD-CM is a clinical research training fellow of the Medical Research Council (UK). The Southwark and Lambeth pulmonary rehabilitation team is in part funded by "Pursuing Perfection," coordinated by the NHS Modernisation Agency

Competing interests: None declared.

Ethical approval: The local research ethics committee approved the study.

1 British Lung Foundation. Lung report III-casting a shadow over the nation's health London: British Lung Foundation, 2003.

2 Garcia-Aymerich J, Farrero E, Felez MA, Izquierdo J, Marrades RM, Anto JM. Risk factors of readmission to hospital for a COPD exacerbation: a prospective study. Thorax 2003;58:100-5.

3 Griffiths TL, Burr ML, Campbell IA, Lewis-Jenkins V, Mullins J, Shiels K, et al. Results at 1 year of outpatient multidisciplinary pulmonary rehabilitation: a randomised controlled trial. Lancet 2000;355:362-8. 
4 Lacasse Y, Brosseau L, Milne S, Martin S, Wong E, Guyatt GH, et al. Pulmonary rehabilitation for chronic obstructive pulmonary disease. Cochrane Database Syst Rev 2002(3):CD003793

5 National Institute for Clinical Excellence (NICE). Chronic obstructive pulmonary disease: national clinical guideline for management of chronic obstructive pulmonary disease in adults in primary and secondary care. Thorax 2004;59(suppl 1).

6 Singh SJ, Morgan MD, Scott S, Walters D, Hardman AE. Development of a shuttle walking test of disability in patients with chronic airways obstruction. Thorax 1992;47:101924.

7 Jones PW, Quirk FH, Baveystock CM, Littlejohns P. A self-complete measure of health status for chronic airflow limitation. The St. George's respiratory questionnaire. Am Rev Respir Dis 1992;145:1321-7.

8 Guyatt GH, Berman LB, Townsend M, Pugsley SO, Chambers LW. A measure of quality of life for clinical trials in chronic lung disease. Thorax 1987;42:773-8.

9 Ware JE Jr, Sherbourne CD. The MOS 36-item short-form health survey (SF-36). I Conceptual framework and item selection. Med Care 1992;30:473-83

10 Seemungal TA, Donaldson GC, Bhowmik A, Jeffries DJ, Wedzicha JA. Time course and recovery of exacerbations in patients with chronic obstructive pulmonary disease. $A m J$ Respir Crit Care Med 2000;161:1608-13.

11 Spencer S, Jones PW. Time course of recovery of health status following an infective exacerbation of chronic bronchitis. Thorax 2003;58:589-93.

12 Aaron SD, Vandemheen KL, Hebert P, Dales R, Stiell IG, Ahuja J, et al. Outpatient oral prednisone after emergency treatment of chronic obstructive pulmonary disease. $N$ Engl J Med 2003;348:2618-25.

13 Man WD, Soliman MG, Nikoletou D, Harris ML, Rafferty GF, Mustfa N, et al. Non-volitional assessment of skeletal muscle strength in patients with chronic obstructive pulmonary disease. Thorax 2003;58:665-9.

14 Spruit MA, Gosselink R, Troosters T, Kasran A, Gayan-Ramirez G, Bogaerts P, et al. Muscle force during an acute exacerbation in hospitalised patients with COPD and its relationship with CXCL8 and IGF-I. Thorax 2003;58:752-6.
15 Bourbeau J, Julien M, Maltais F, Rouleau M, Beaupre A, Begin R, et al. Reduction of hospital utilization in patients with chronic obstructive pulmonary disease: a disease-specific self-management intervention. Arch Intern Med 2003;163:585-91.

16 Behnke M, Taube C, Kirsten D, Lehnigk B, Jorres RA, Magnussen H. Home-based exercise is capable of preserving hospital-based improvements in severe chronic obstructive pulmonary disease. Respir Med 2000;94:1184-91.

17 Britton M. The burden of COPD in the U.K.: results from the confronting COPD survey. Respir Med 2003;97(suppl C):S71-9.

18 Griffiths TL, Phillips CJ, Davies S, Burr ML, Campbell IA. Cost effectiveness of an outpatient multidisciplinary pulmonary rehabilitation programme. Thorax 2001;56:77984

(Accepted 7 September 2004)

doi $10.1136 / \mathrm{bmj} .38258 .662720 .3 \mathrm{~A}$

Respiratory Muscle Laboratory, Guy's, King's, and St Thomas' School of Medicine, King's College Hospital, London SE5 9PJ

William D-C Man MRC clinical research fellow

John Moxham professor of respiratory medicine

King's College Hospital, London SE5 9RS

Nora Donaldson senior lecturer in statistics

Barry J Gray consultant physician in respiratory medicine

Royal Brompton Hospital, London SW3 6NP

Michael I Polkey consultant physician in respiratory medicine

Correspondence to: W D-C Man william.man@kcl.ac.uk 